\title{
Uso del WhatsApp en Comunidades Interactivas de Aprendizaje: Perspectiva Estudiantil sobre Ejercicios de Pronunciación en la Clase de Inglés para Propósitos Específicos
}

Karol Cubero y Lucia Villanueva

Universidad Nacional de Costa Rica

\begin{abstract}
The Internet, the multiplicity of tools and applications on a mobile device have transformed different areas of life today and education is no exception. Mobile learning is acquiring a growing relevance in academic and educational spaces. This article describes a learning session by implementing WhatsApp to facilitate activities to practice English pronunciation. With a sample of 30 students, mostly between the age of 20 to 25 years, they were the participants of a class session facilitated by the use of WhatsApp, based on pronunciation exercises with specific tourism content, addressed to these students who belong to the Sustainable Tourism Business Management major.
\end{abstract}

Keywords: WhatsApp, pronunciation, learning communities, mobile learning, formative evaluation.

\section{Resumen}

Internet y la multiplicidad de herramientas y aplicaciones accesibles en un dispositivo móvil se han convertido en multifacéticas herramientas en todos los ámbitos de la vida actual y el ambiente educativo no es la excepción. El aprendizaje móvil esta adquiriendo cada vez mayor relevancia en espacios académicos y formativos. El presente trabajo describe una sesión de aprendizaje haciendo uso de la aplicación WhatsApp para mediar actividades en el idioma Inglés. Se trabajó con una muestra de 30 estudiantes con edades, en su mayoría, entre 20 y 25 años, a quienes se les facilitó, mediante la aplicación del WhatsApp, actividades enfatizadas en ejercicios de pronunciación con contenido turístico específico, dirigidos a estudiantes pertenecientes a la carrera de Gestión Empresarial del Turismo Sostenible.

Palabras clave: WhatsApp, pronunciación, comunidades de aprendizaje, aprendizaje móvil, evaluación formativa.

\section{Introducción}

En los días que vivimos, es inminente el uso de los teléfonos celulares y sus aplicaciones tecnológicas incorporadas. Cada vez más dependemos de este aparato por las facilidades que nos presenta en múltiples tareas desde las más cotidianas hasta las más complejas, con fines 
Uso del WhatsApp en Comunidades Interactivas de Aprendizaje: Perspectiva Estudiantil sobre Ejercicios de Pronunciación en la Clase de Inglés para Propósitos Específicos

académicos de aprendizaje o no. Entonces, su incorporación al campo educativo es un hecho ineludible que los docentes debemos aplicar y al que nos obliga a estar informados e investigar al respecto para sacarles el mejor provecho con propósitos de aprendizaje. Si para nuestros estudiantes e incluso para los docentes mismos es difícil apartarse de este aparato inteligente, ¿porqué no hacer de él una herramienta útil y un compañero más en los propósitos educativos? Las tecnologías cambian vertiginosamente y de igual manera sus posibles beneficios o limitaciones. Es por ello que los docentes debemos estar actualizados y en constante investigación de las mismas para ser aplicadas como herramienta educativa. Esta es una invitación constante. En este sentido, es que la presente investigación busca como docentes de inglés como lengua extranjera, analizar el aprovechamiento de la aplicación de WhatsApp, basándose en la motivación e interés demostrada por los estudiantes hacia sus teléfonos celulares y en este caso, estudiando uno de las habilidades de la lengua como lo es la pronunciación. Precisamente, la pronunciación del inglés es una dificultad presente en muchos de los estudiantes y no es, muchas veces, abordada de la mejor manera en el aula. Las bondades que presenta esta aplicación permite su práctica con materiales auténticos y en tiempo real, lo que favorece la interacción y la cooperación entre pares con una evaluación cualitativa.

\section{Objetivos de la Propuesta}

\section{Objetivo General:}

Identificar perspectivas estudiantiles sobre el uso del WhatsApp en el aprendizaje del Idioma Inglés para propósitos específicos mediante actividades y ejercicios de pronunciación aplicada a estudiantes de la carrera de gestión empresarial del Turismo Sostenible de la UNA, Sede Regional Chorotega, Guanacaste

\subsection{Sustentos Teóricos}

\subsubsection{Aprendizaje móvil}

Según Brazuelo F. y Gallego D. (2011), el aprendizaje móvil se describe como la modalidad educativa que facilita la construcción del conocimiento, la resolución de problemas de aprendizaje y el desarrollo de destrezas o habilidades diversas de forma autónoma y ubicua gracias a la mediación de dispositivos móviles portables. Al respecto, Hockly y Dudeney (2014) proponen una serie de actividades basadas en el uso del teléfono celular entre las cuales se encuentran actividades básicas basadas en simples mensajes de texto a otras ya más complejas. Ellos establecen que los teléfonos celulares son excelentes herramientas para ayudar a los estudiantes a producir el idioma meta, pero advierten que los profesores deben siempre de preguntarse porque se esta implementando el aprendizaje móvil y asi tener claridad en lo que se desea alcanzar. Otras opiniones también positivas respecto a esta metodología como herramienta de soporte en las clases de inglés nos la ofrecen Thornton, y Houser (2005) al destacar que gracias a la innovación tecnológica, el aprendizaje móvil se convertirá en el centro de atención en educación en un futuro próximo ya que las 
posibilidades y facilidades de usar una herramienta como el teléfono móvil amplia las oportunidades de que los alumnos aprendan de forma significativa (p. 49)

\subsubsection{La aplicación de WhatsApp en la mediación de aprendizajes}

La aplicación de WhatsApp es una multiplataforma de mensajería que permite enviar y recibir mensajes sin pagar por SMS ya que utiliza los datos móviles ya obtenidos. Esta aplicación resulta muy atractiva por sus diversas características, primeramente porque es gratuita y luego porque se pueden crear grupos, resulta fácil de usar y desde esta se pueden enviar un número ilimitado de imágenes, vídeos y mensajes de audio promoviendo asi una interacción en tiempo real. Esta aplicación ha sido sumamente exitosa durante los últimos años pues ha captado la atención de millones de usuarios entre estos académicos, docentes e investigadores. Hidalgo (2013) sostiene que los alumnos ya lo usan en entornos educativos sin que ningún profesor les haya propuesto hacerlo. Los usos académicos que los estudiantes le dan es principalmente la creación de grupos para ponerse de acuerdo en trabajos en equipo que mandan en clase y consultar dudas a los compañeros.

Ademas, este permite activar y promover redes de aprendizaje los que se conocen como entornos de aprendizaje en línea que ayudan a los participantes a desarrollar sus competencias colaborando y compartiendo información. En ese sentido, Koper, 2009 define que las redes de aprendizaje están diseñadas para tratar de enriquecer la experiencia de aprendizaje en los contextos de educación desde las cuales se intercambian experiencias y conocimiento con otros, se brinda y recibe apoyo de otros participantes, se promueve diferentes tipos de evaluación.

1.1.3 Ventajas en el uso del WhatsApp en la mediación del aprendizaje del Inglés

Muchos investigadores concuerdan a nivel general que el uso del WhatsApp en la educación puede ser positivo si existe un claro planteamiento de objetivos académicos y se destaca las siguientes ventajas:

- Promueve un un entorno motivador y lúdico, ofrece la posibilidad de trabajar en tiempo y contexto real, las respuestas son espontáneas, permite la exposición inmediata al idioma meta mediante la interacción y la cohesión grupal.

- Potencia la construcción activa y colectiva del aprendizaje, la herramienta, se puede integrar como banco de material educativo alternativo y complementario. También facilita la adquisición de la competencia en la búsqueda y selección de la información en la que los educandos pueden indagar e investigar en la web materiales educativos significativos sobre los contenidos que se ven en clase.

- Facilita el pensamiento crítico ente el alumnado mediante la propuesta de preguntas que generen debate y diálogo acerca de los contenidos de clase.

- Realizar diagnósticos sobre los conocimientos previos de los estudiantes acerca de contenidos.

- Realizar evaluaciones de seguimiento sobre la adquisición del conocimiento entre el alumnado fomentando la re-elaboración de su propio conocimiento para comunicarlo. 
Uso del WhatsApp en Comunidades Interactivas de Aprendizaje: Perspectiva Estudiantil sobre Ejercicios de Pronunciación en la Clase de Inglés para Propósitos Específicos

- De igual forma Barhoumi Chokri (2015) destaca que el WhatsApp facilita la socialización de productos de aprendizaje en la que los estudiantes cooperan, colaboran y participan en las actividades propuestas.

- En relación al aprendizaje del Inglés, Colon et al. (2012) manifiestan las posibilidades del aprendizaje mediante el uso de WhatsApp en el desarrollo de habilidades de lectura mediante el envío de breves mensajes, plantean que los estudiantes se sintieron motivados hacia la lectura en Inglès.

- Se brinda la posibilidad al alumnado de interactuar a diario en la lengua meta lo que ha supuesto un estímulo a los educandos para ganar agilidad en la lengua inglesa así como perder el miedo al usar el idioma

\subsubsection{Desventajas en el uso de WhatsApp en la mediación de aprendizajes}

Autores e investigadores que han llevado a cabo estudios e investigaciones sobre la temática, han encontrado dificultades durante la realización de sus proyectos. A continuación, se puntualizan algunas de las principales dificultades encontradas.

Gutiérrez-Colon et al. (2012) mencionan en su proyecto que a los participantes inicialmente no les agradaría recibir mensajes académicos una vez terminado su horario de la facultad. En cambio, una vez finalizada la investigación se concluyó que los alumnos no percibieron esta metodología como intrusiva.

Otra desventaja a la que apuntan varios autores por ejemplo Kennedy et al. (2008) y Thornton et al. (2005), es el hecho que las pantallas de los teléfonos móviles son muy pequeñas. Por lo tanto, hay que crear ejercicios adecuados a la medida de las pantallas para que las actividades estén especialmente diseñadas para el aprendizaje móvil y no se hagan tediosas o pesadas de leer.

\subsection{5 ¿Porqué enseñar pronunciación?}

Al estudiar cualquier lengua extranjera se hace fundamental estudiar su pronunciación. La pronunciación es un elemento esencial dentro de los componentes de la habilidad de la conversación y que aunado al manejo idóneo de las otras habilidades como la comprensión auditiva, la escritura y la lectura, demuestran cuan proficiente un individuo es en la lengua extranjera en cuestión. Es así como la práctica de la pronunciación se vuelve muy importante para poder comunicarse efectivamente e interactuar utilizando la lengua meta, convirtiéndose, indiscutiblemente, en la delimitación de la competencia comunicativa del hablante.

De acuerdo a nuestra experiencia como profesoras de lengua extranjera, en este caso de inglés, hemos podido observar las dificultades que tienen los estudiantes al momento de pronunciar este idioma extranjero y esto, principalmente, porque el idioma inglés difiere del español en muchos aspectos pero en especial, porque la mayoría de las palabras en inglés no se pronuncian como se escriben y también porque hay sonidos de ese idioma que no existen en español. Esto representa una mayor dificultad para los hispano hablantes que desean hablar inglés y por ello es causa de frustración en el proceso de aprendizaje de muchos estudiantes.

(cc) EY-NC-ND 2017, Universitat Politècnica de València 
El estudio de la pronunciación en el aula, entonces, es un gran reto, no solo para el estudiante sino también para el profesor quien tiene que aplicar las estrategias más convenientes para ayudar a sus aprehendientes a adquirir la pronunciación correcta del idioma. La realidad, en muchas ocasiones, es que no se le da el tiempo suficiente para enseñar en el aula aspectos referentes a la pronunciación y en muchos casos, ni siquiera se contempla la pronunciación como un contenido específico de estudio dentro del programa académico. En otros casos, la pronunciación se enseña de manera aislada, sin darle oportunidad al estudiante de percibir esta competencia de la lengua de manera integrada, con todos sus componentes. Este panorama complica el manejo eficiente del idioma por cuanto , si no se tiene la pronunciación correcta, el hablante no podrá darse a entender en su sentido correcto y completo, lo que se prestaría a verdaderos mal entendidos. Si existe una carencia en este sentido, no solo afecta la comunicación oral, sino que perjudica también las otras habilidades de la lengua ya mencionadas como la lectura, la comprensión auditiva y hasta la escritura.

\subsubsection{Elementos de la pronunciación}

La Real Academia de la Lengua Española define el término pronunciar como la acción de emitir y articular sonidos. Iruela (2003) explica la importancia de la pronunciación en la función comunicativa de la lengua cuando hace referencia a la composición de la competencia comunicativa de acuerdo al Marco Común Europeo de Referencia para las Lenguas (MCER). En este sentido, la competencia comunicativa según MCER se compone de competencias lingüísticas, pragmáticas y socioculturales. Dentro de las competencias lingüísticas se encuentran, a su vez, otras categorías o grupos como los son las competencias referentes a la léxica, a la gramática, a la semántica, a la fónica, ortográfica y la ortoépica. Cada una de estas competencias se vinculan entre sí pero en especial la competencia fónica que está unida, por ejemplo, con la léxica al considerar que un individuo ha adquirido una palabra cuando la puede pronunciar con claridad y la reconoce de manera auditiva. O bien, en el caso de la ortoépica que es la competencia referida a leer en voz alta y de forma correcta un texto o verso con solo conocer las reglas de pronunciación. Todo lo anterior, ilustra las interrelaciones entre los diferentes componentes de la competencia comunicativa, su complejidad y dinamismo y en el que la pronunciación juega un papel relevante. Iruela (2003).

\subsubsection{Enseñanza de la Pronunciación}

En lo referente a los componentes de la pronunciación, Wei (2006) destaca la necesidad de dejar de lado viejas prácticas en cuanto a la enseñanza de la pronunciación de una lengua extranjera e ir más allá de la enseñanza basada en la discriminación de sonidos, contrastes de sonidos en palabras, vocales y consonantes. El estudio de la pronunciación en el aula comprende un conjunto de componentes. Para Scarcella y Oxford (1994) citado por Wei (2006), esos componentes son los siguientes:

Entonación: Se refiere al tono en la que un sonido de voz es pronunciado. Una buena entonación nunca sonara monótono o aburrido.

Acento y ritmo: Muy relacionados ya que el acento contribuye al ritmo. El acento o énfasis en una sílaba, en una oración o conexiones, produce el ritmo o música de la lengua. En inglés el ritmo, por lo general, ocurre en intervalos regulares. 
Uso del WhatsApp en Comunidades Interactivas de Aprendizaje: Perspectiva Estudiantil sobre Ejercicios de Pronunciación en la Clase de Inglés para Propósitos Específicos

Consonantes: Se refiere a los sonidos producidos o pronunciados con un bloqueo del paso del aire. Lo que se estudia en este aspecto es la sustitución, omisión, articulación, conexiones o links y clusters.

Vocales: Al contrario de las consonantes, las vocales se pronuncian con el paso libre del aire. Se estudia de las vocales aspectos como sustitución, articulación, duración, reducción y enlaces o links.

En cuanto a los enfoques de enseñanza de la pronunciación, los mismo han ido evolucionando en los últimos 35 años y hoy se pueden encontrar diferencias en los enfoques tradicionales y en los que están en tendencia.

Scarcella y Oxford (1994) citado por Wei (2006) realizan una comparación entre un enfoque de enseñanza tradicional de la pronunciación y un enfoque basado en la investigación o "Research Based Approach" y en la que se destacan diferencias importantes como que en el enfoque basado en la investigación, prive la necesidad de aprender pronunciación para darse a entender en el idioma extranjero. No se busca entonces, la imitación de acentos nativos sino que se respetan los acentos propios del aprehendiente y los mismos son valorados como carácter e identidad cultural. Esto se une a una enseñanza integral en donde se estudia el ritmo y la entonación a la vez que se motiva al estudiante, quien es el centro de su propio aprendizaje.

Tabla 1. Comparación de enfoques en la enseñanza de la pronunciación

\begin{tabular}{|l|l|}
\hline Enfoque Basado en investigación & Enfoque tradicional \\
\hline $\begin{array}{l}\text { El objetivo es ganar suficientes habilidades en la } \\
\text { pronunciación para que la calidad de la } \\
\text { pronunciación no inhiba la comunicación }\end{array}$ & $\begin{array}{l}\text { El objetivo de la instrucción es adquirir una } \\
\text { pronunciación nativa. }\end{array}$ \\
\hline $\begin{array}{l}\text { En vez de enfatizar en los sonidos, los profesores } \\
\text { se concentran en el stress y la entonación. }\end{array}$ & El énfasis primario es enseñar sonidos. \\
\hline $\begin{array}{l}\text { El énfasis de la instrucción es enseñar la } \\
\text { pronunciación con un enfoque comunicativo }\end{array}$ & $\begin{array}{l}\text { Los sonidos se enseñan no con el enfoque } \\
\text { comunicativo mediante ejercicios o "drills" } \\
\text { aislados. }\end{array}$ \\
\hline $\begin{array}{l}\text { El profesor provee a los estudiantes de } \\
\text { transcripciones fonéticas solo cuando ellas son } \\
\text { útiles para los estudiantes en tutorías. }\end{array}$ & $\begin{array}{l}\text { Transcripciones fonéticas son componentes } \\
\text { básicos de las clases tradicionales de } \\
\text { pronunciación. }\end{array}$ \\
\hline $\begin{array}{l}\text { La motivación de los estudiantes es básica para } \\
\text { aprender exitosamente el idioma. El estudiante } \\
\text { juega un papel primordial para el mejoramiento } \\
\text { de su propia pronunciación. Se enseñan } \\
\text { estrategias de auto monitoreo y de auto atención. }\end{array}$ & $\begin{array}{l}\text { Estudiantes no toman responsabilidad por } \\
\text { el mejomiento de su } \\
\text { pronunciación. }\end{array}$ \\
\hline $\begin{array}{l}\text { La estima o afecto es crucial en la enseñanza de } \\
\text { la pronunciación. Estudiantes aprenden técnicas } \\
\text { de relajación para bajar los niveles de ansiedad y } \\
\text { resistencia a mejorar la pronunciación. }\end{array}$ & $\begin{array}{l}\text { La estima o afecto no se considera como } \\
\text { una importante actividad instructiva }\end{array}$ \\
\hline
\end{tabular}

\section{Fuente: Wei (2006)}

Igualmente, para Scarcella y Oxford (1994) citado por Wei (2006) establecen técnicas de enseñanza de la pronunciación bajo el enfoque no tradicional de investigación en las cuales destacan las que refuerzan el auto monitoreo, el estudio por cuenta propia, corrección individual y uso de la tecnología, entre otros aspectos.

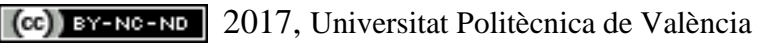


Tabla 2. Técnicas de enseñanza de la pronunciación bajo el enfoque investigativo

\begin{tabular}{|c|c|}
\hline Técnicas & Contenidos \\
\hline Auto monitoreo & Aprenden a auto monitorearse para mejorar su inteligibilidad \\
\hline $\begin{array}{l}\text { Sesiones tutoriales de } \\
\text { estudio individual }\end{array}$ & $\begin{array}{l}\text { Empieza con un diagnóstico de cada estudiante y se le diseña al } \\
\text { estudiante un programa personalizado }\end{array}$ \\
\hline Corrección Individual & $\begin{array}{l}\text { Reporte de los resultados de cada producción del estudiante de manera } \\
\text { individual. }\end{array}$ \\
\hline $\begin{array}{l}\text { Actividades } \\
\text { Comunicativas }\end{array}$ & Diseñadas para que los estudiantes practiquen sonidos específicos. \\
\hline $\begin{array}{l}\text { Versiones escritas de } \\
\text { presentaciones orales }\end{array}$ & $\begin{array}{l}\text { En niveles más avanzados, se les puede entregar a los estudiantes las } \\
\text { estrategias para el análisis de la versión escrita de su propia producción } \\
\text { oral. }\end{array}$ \\
\hline $\begin{array}{ll}\text { Asistencia en } \\
\text { computadora de } \\
\text { aprendizaje del idioma }\end{array}$ & $\begin{array}{l}\text { Profesor utiliza apoyos visuales de patrones de discurso para enseñar } \\
\text { entonación, stress, y sonidos de manera grupal o individual. }\end{array}$ \\
\hline Explicaciones & $\begin{array}{l}\text { Explicaciones de cómo se producen los sonidos deben mantenerse al } \\
\text { mínimo, aunque las instrucciones sobre qué hacer con los órganos } \\
\text { vocales sí puede ayudar bajo ciertas circunstancias. }\end{array}$ \\
\hline $\begin{array}{l}\text { Utilización de sonidos } \\
\text { conocidos }\end{array}$ & $\begin{array}{l}\text { Comparación con la primera lengua del estudiante puede ayudarlo a } \\
\text { producir la lengua meta. }\end{array}$ \\
\hline $\begin{array}{l}\text { Incorporación de nuevos } \\
\text { elementos }\end{array}$ & Con dirección o ayuda del profesor \\
\hline $\begin{array}{l}\text { Estrategias } \\
\text { comunicativas }\end{array}$ & $\begin{array}{l}\text { Los estudiantes pueden aprender algunas estrategias comunicativas para } \\
\text { darles la impresión de que su pronunciación es mejor de lo que realmente } \\
\text { pueda estar como metodología de recuperación, práctica para la } \\
\text { comunicación. }\end{array}$ \\
\hline Estrategias afectivas & Para disminuir la ansiedad y ganar confianza. \\
\hline
\end{tabular}

Fuente: Wei (2006)

Cheng (1998) citado por Wei (2006) recomienda, diversas estrategias para enseñar pronunciación. Algunas de ellas son proveer material significativo como artículos y lecturas para practicar ritmo, acento o stress, entonación, "linkings" o enlaces. A su vez, el uso de canciones, juegos y trabalenguas, incrementa la motivación y es divertido para los estudiantes. Muy importante también es el evaluar el progreso de los estudiantes para mantener una buena motivación en ellos. En este último aspecto, pesa mucho la manera en que se evalúa. La evaluación cualitativa es una muy recomendable opción para mantener a los estudiantes motivados e interesados y más aún cuando la evaluación se realiza entre pares. Por ello, a continuación, se explica aspectos relevantes relacionados con la evaluación cualitativa.

\subsubsection{La evaluación cualitativa de los aprendizaje}

La evaluación cualitativa ha generado cierto agrado y resonancia en los últimos años, al proponer un proceso de enseñanza-aprendizaje desde niveles más integrales y holístico. Se dice holístico porque toma en cuenta varios elementos que intervienen en ella: los objetivos, procesos, métodos, recursos, contexto e instrumentos. Al respecto Alfaro (1996) expresa que la evaluación cualitativa se percibe como un proceso de investigación integral, la cual aporta 
Uso del WhatsApp en Comunidades Interactivas de Aprendizaje: Perspectiva Estudiantil sobre Ejercicios de Pronunciación en la Clase de Inglés para Propósitos Específicos

evidencias diarias de la participación del estudiante en la práctica académica. Esta evaluación acentúa la importancia de observar e interpretar situaciones y experiencias, más que en resultados numéricos y situaciones controladas como lo hace la evaluación cuantitativa. La evaluación cualitativa invita a la evaluación permanente, con el objetivo de retroalimentar y reorientar a lo largo del proceso educativo. Para lograr mediar estas prácticas, es necesario plantearse estrategias e instrumentos que respondan a cómo evaluar.

\subsubsection{Estrategias e Instrumentos de Evaluación Cualitativa}

Bordas y Cabrera (2001) afirman que las estrategias de evaluación planeadas con el fin de recopilar información para reforzar el aprendizaje son determinantes en el proceso de enseñanza-aprendizaje, por tanto, generan un aprendizaje profundo, desarrollador de habilidades de autoconocimiento y autorregulación en un contexto participativo. Las estrategias de evaluación pueden ser muy variadas, entre estas: los diarios reflexivos, portafolios, la entrevista, el estudio de caso, panel, exposiciones, entre otras. Estas estrategias se refuerzan utilizando instrumentos como las rúbricas, que favorecen una evaluación centrada en el proceso. Estas son herramientas que se utilizan para emitir criterios sobre el desempeño del estudiante en áreas específicas. Dichos criterios son los parámetros a utilizar para ayudar al estudiante a mejorar. De hecho, los criterios para calificar un área en específico se dan a conocer previamente a los estudiantes y contribuir así para su mejor preparación. Las rúbricas se aplican tanto para el alumno como para el profesor y se dan de manera auto evaluativa, de pares o grupal.

\subsection{9 ¿Por qué aplicar la evaluación cualitativa?}

La enseñanza aprendizaje desde el enfoque humanista, pretende revalorizar a las personas como actores activos, al considerar todas sus particularidades e individualidades y promover un profundo autoconocimiento, un reencuentro con su integridad. Al hablar de proceso formativo consideramos también el proceso evaluativo. El valor humanístico que se pueda agregar a la evaluación reside en respetar y potencializar esa integridad, atendiendo diferencias, sean estas culturales, étnicas, religiosas o personales. Los educadores deben de hacer de la evaluación cualitativa una práctica continua, bajo procedimientos sistematizados que consideren no solo los aspectos cognitivos, sino también los socio-afectivos, las habilidades, ritmo de aprendizaje y el contexto de los estudiantes. Durante el planeamiento de una evaluación integral, las actividades de aprendizaje son insumos importantes en la valoración del proceso de aprendizaje, en donde se reconocen las fortalezas y las debilidades por medio de la retroalimentación. Esta dinámica permite abrir espacios de intercambio que reorienten, ajusten y mejoren el proceso, según el logro de los objetivos planteados a nivel individual y colectivo. La práctica continua hace que los estudiantes vayan perdiendo el temor a verse y sentirse evaluados, pues experimentan seguridad, creatividad y respeto. Igualmente, a nivel colectivo, la evaluación cualitativa fomenta valores como la solidaridad, cooperación y comunicación.

(c)) EY-NC-ND 2017, Universitat Politècnica de València 
En nuestra experiencia como profesoras de un idioma extranjero, hemos verificado los beneficios de la evaluación cualitativa al evaluar estudiantes en el desempeño específico de un idioma, esto no solo en el aspecto cognitivo, sino afectivo. Esta concepción es un elemento motivador para el estudiante, pues fomenta el aprender a aprender, lo cual contribuye a formar la autonomía y autoestima del estudiante en un ambiente lleno de experiencias agradables y amigables.

\subsection{Propuesta: Mediación de aprendizaje basada en ejercicios de pronunciación en una clase de Inglés para Propósitos Específicos}

A continuación se detalla la propuesta utilizada en la mediación de los aprendizajes en dos cursos de Inglés con propósitos específicos. Se exponen los objetivos y la descripción de las actividades realizadas con la finalidad de determinar el grado de efectividad de implementar el aprendizaje móvil mediante la aplicación del WhatsApp para realizar prácticas de pronunciación del idioma Inglés.

- Identificar la efectividad de la aplicación del WhatsApp en la mediación de actividades relacionadas con la pronunciación del idioma Inglés.

- Mediar, a través de comunidades de aprendizaje en línea desde la aplicación WhatsApp, actividades de pronunciación y de contenido especifico con instrucciones claras para guiar a los educandos en la práctica del acento, entonación, ritmo y sonidos del idioma Inglés.

- Promover en las sesiones, el aprendizaje colaborativo mediante WhatsApp y una transparente realimentación facilitada por los mismos participantes de la comunidad (grupo de WhatsApp) mediante una rúbrica de evaluación facilitada por las mediadoras.

Temas: Acento, entonación, ritmo y sonidos finales de verbos pasados regulares

Materiales: Teléfono inteligente, Acceso a Internet, lectura y dialogo en audio formato mp3 pregrabado, aplicación de WhatsApp, audífonos, rúbrica de evaluación pronunciación.

\section{Procedimientos:}

1. Informar a los estudiantes los objetivos de la implementación del aprendizaje móvil en algunas sesiones de clases.

2. Realizar comunidades de aprendizaje( grupos de WhatsApp),organizando el grupo de educandos en pequeños grupos de comunidades de aprendizaje de máximo 10 estudiantes.

3. Se solicita a los estudiantes agruparse en grupos según la comunidad de aprendizaje que integra. Una vez listos se remiten las instrucciones vía WhatsApp de la actividad 1.

Actividad 1:Enviar mediante la aplicación de WhatsApp, las instrucciones y la actividad a realizar tendientes a practicar algunos aspectos de pronunciación. Instrucción enviada al WhatsApp: Escuchen el audio grabado por la profesora, este contiene un extracto de un 
Uso del WhatsApp en Comunidades Interactivas de Aprendizaje: Perspectiva Estudiantil sobre Ejercicios de Pronunciación en la Clase de Inglés para Propósitos Específicos

articulo sobre trabajos turísticos, verifiquen comprensión y dirijan su atención a patrones de pronunciación, entonación, acento, ritmo y sonidos finales del idioma Inglés.

- Se adjunta el audio con la lectura que deben escuchar y de la cual se realizarán las actividades requeridas.

- Se solicita a los estudiantes que reproduzcan la lectura y que tomen unos minutos para su comprensión.

- Una vez que clarifican compresión, se les solicita que se graben, imitando y procurando similitud en los aspectos de pronunciación destacados, entonación, acento, ritmo y sonidos lo mas similares a la pronunciación del audio clip.

- El docente guía a los estudiantes en la realización de sus productos, monitoreando sus practicas de repetición y apoyándoles en algunas dificultades emergentes.

- Al terminar su grabaciones, los estudiantes se evalúan formativamente entre si mediante una rubrica de evaluación que se reparte al terminar sus productos. Luego envían su realimentación al WhatsApp, donde en sus comunidades de aprendizaje se extiende la oportunidad de mejorar y valorar sus fortalezas y debilidades en la pronunciación del inglés.

Actividad 2: Enviar mediante la aplicación de WhatsApp, las instrucciones y la actividad realizar tendiente a practicar algunos aspectos de pronunciación. Instrucción enviada al WhatsApp: Escuchen el audio, este contiene un dialogo describiendo una visita a un lugar turístico. Verifiquen comprensión y dirijan su atención a patrones de pronunciación, entonación, acento, ritmo y sonidos finales del idioma Inglés

- Se adjunta el audio con el dialogo que deben escuchar y del cual se realizará la actividad requerida.

- Se solicita a los estudiantes, formar parejas desde su comunidad de aprendizaje en linea, que reproduzcan el dialogo las veces que requieran para su comprensión.

- Una vez que clarifican compresión, se les solicita que se graben realizando el dialogo, imitando y procurando similitud en los aspectos de pronunciación destacados, entonación, acento, ritmo y sonidos lo mas similares a la pronunciación del audio clip.

- Durante las grabaciones de ambas actividades, el docente monitorea el desempeño de los estudiantes, observando su actitud hacia la mediación de las actividades desde el WhatsApp.

- Asi mismo se guía a los estudiantes en la realización de sus productos, mediante practicas de repetición constantes.

- Al terminar su grabaciones, los estudiantes se evalúan formativamente entre si mediante una rubrica de evaluación que se reparte al terminar sus productos. Al evaluarse formativamente, guiados con la rubrica de evaluación formativa, se comparten los comentarios motivando la realimentación de pares que consideren pertinente destacar tanto a nivel de debilidades como fortalezas.

(cc) EY-NC-ND 2017, Universitat Politècnica de València 
Estas actividades fueron aplicadas a dos grupos de estudiantes de la Carrera de gestión empresarial del turismo, luego de realizar las actividades mediadas desde el WhatsApp se solicito a los estudiantes contestar un cuestionario con el fin de conocer sus perspectivas sobre la implementación del WhatsApp en la clase para practicar pronunciación del Idioma Inglés.
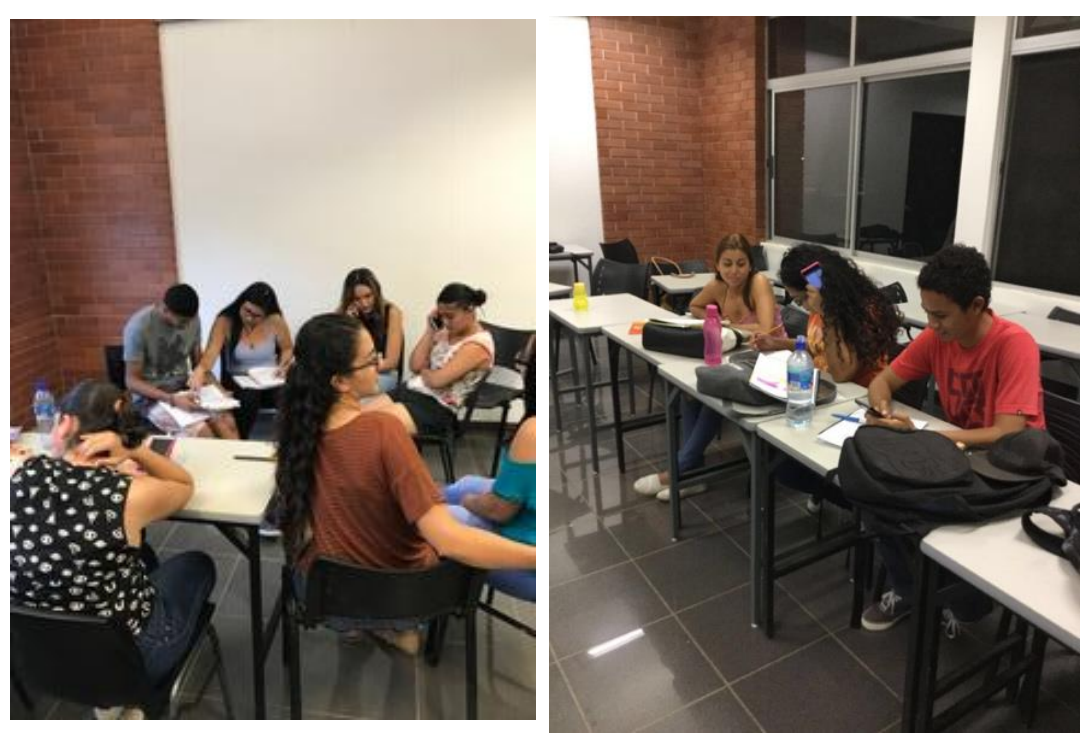

Fig. 1. Fotografías de los estudiantes

\subsubsection{Percepción de los estudiantes sobre el uso del WhatsApp}

Con el objetivo general de identificar las perspectivas de los estudiantes sobre el uso del WhatsApp en el aprendizaje del idioma inglés para propósitos específicos, se realizó una encuesta sobre este aspecto y a continuación se analizan los resultados.

\subsubsection{Objetivos específicos}

- Analizar la frecuencia y posibilidad de uso del WhatsApp.

- Analizar y descubrir la aceptabilidad del whatsApp en la esnseñanza de la pronunciación del inglés.

- Conocer las preferencias y familiaridad de los estudiantes con respecto a la tecnología, redes sociales o aplicaciones electrónicas.

- Motivar al estudiante al aprendizaje, aprovechando las herramientas tecnológicas.

Dentro de la metodología se seleccionaron 30 estudiantes de la carrera de la Gestión Empresarial del Turismo Sostenible de la Universidad Nacional, Sede regional Chorotega, Campus Liberia, 15 de ellos mujeres y 15 hombres con edades que oscilan entre los 20 a 25 años en su gran mayoría.

\subsubsection{Percepción de los estudiantes sobre el uso del whatsApp}

Con el objetivo general de identificar las perspectivas de los estudiantes sobre el uso del WhatsApp en el aprendizaje del idioma inglés para propósitos específicos, se realizó una encuesta sobre este aspecto y a continuación se analizan los resultados. 
Uso del WhatsApp en Comunidades Interactivas de Aprendizaje: Perspectiva Estudiantil sobre Ejercicios de Pronunciación en la Clase de Inglés para Propósitos Específicos

\section{Objetivos específicos}

- Analizar la frecuencia y posibilidad de uso del WhatsApp.

- Analizar y descubrir la aceptabilidad del whatsApp en la esnseñanza de la pronunciación del inglés.

- Conocer las preferencias y familiaridad de los estudiantes con respecto a la tecnología, redes sociales o aplicaciones electrónicas.

- Motivar al estudiante al aprendizaje, aprovechando las herramientas tecnológicas.

Dentro de la metodología se seleccionaron 30 estudiantes de la carrera de la Gestión Empresarial del Turismo Sostenible de la Universidad Nacional, Sede regional Chorotega, Campus Liberia, 15 de ellos mujeres y 15 hombres con edades que oscilan entre los 20 a 25 años en su gran mayoría. ( $80 \%$ de los estudiantes). De estos estudiantes, todos dijeron contar con teléfono celular inteligente siendo el hogar y la universidad los sitios donde suelen conectarse con mayor frecuencia.

Gráfico 1: Tipo de Aparato utilizado para conectarse a internet

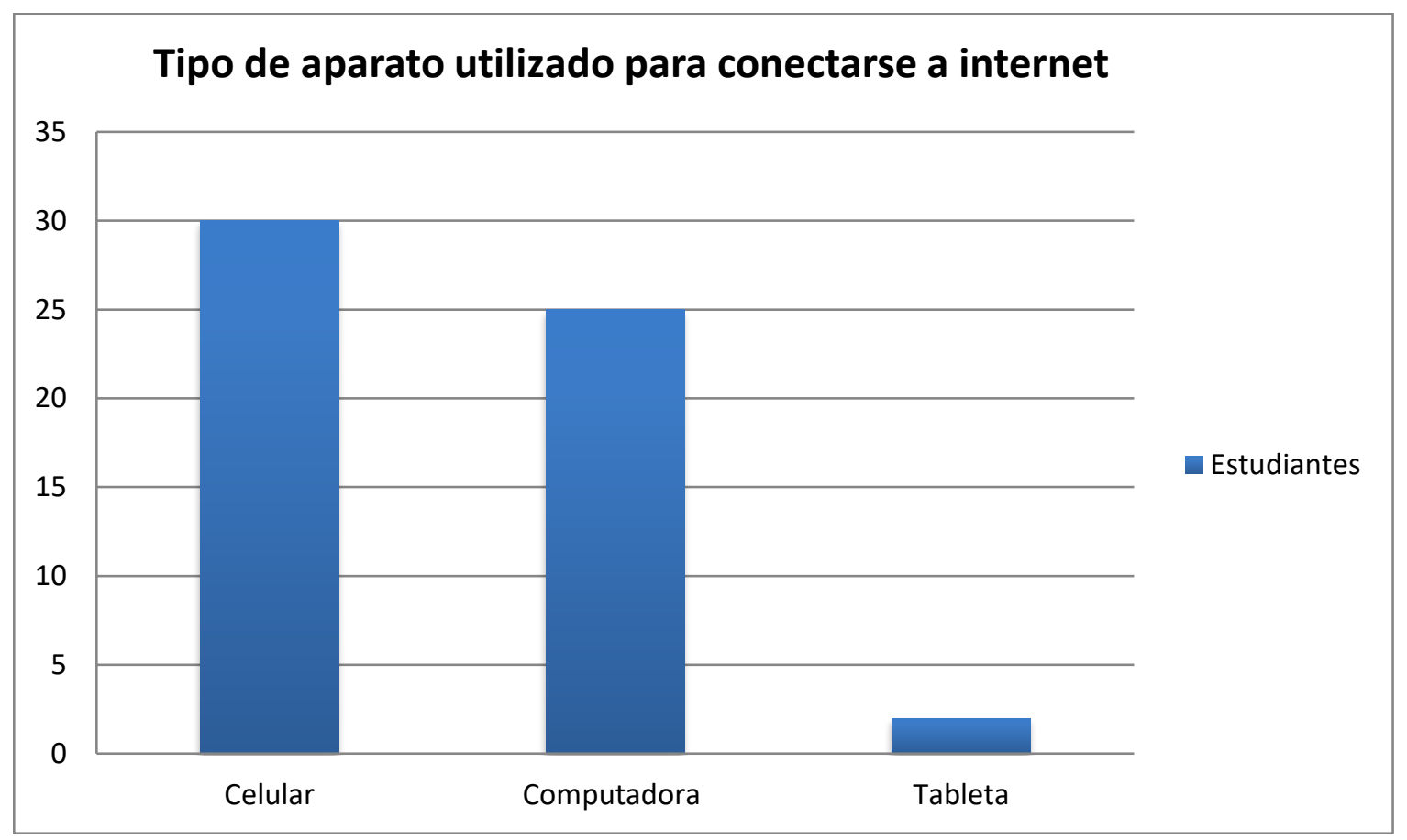

Un $85 \%$ de los estudiantes dijeron utilizar el Internet siempre y casi igual número de estudiantes dijeron contar con algún perfil o cuenta en redes sociales, siendo Facebook el más utilizado seguido por el whatsApp. 
Gráfico 2. Uso de Redes Sociales - perfiles y cuentas

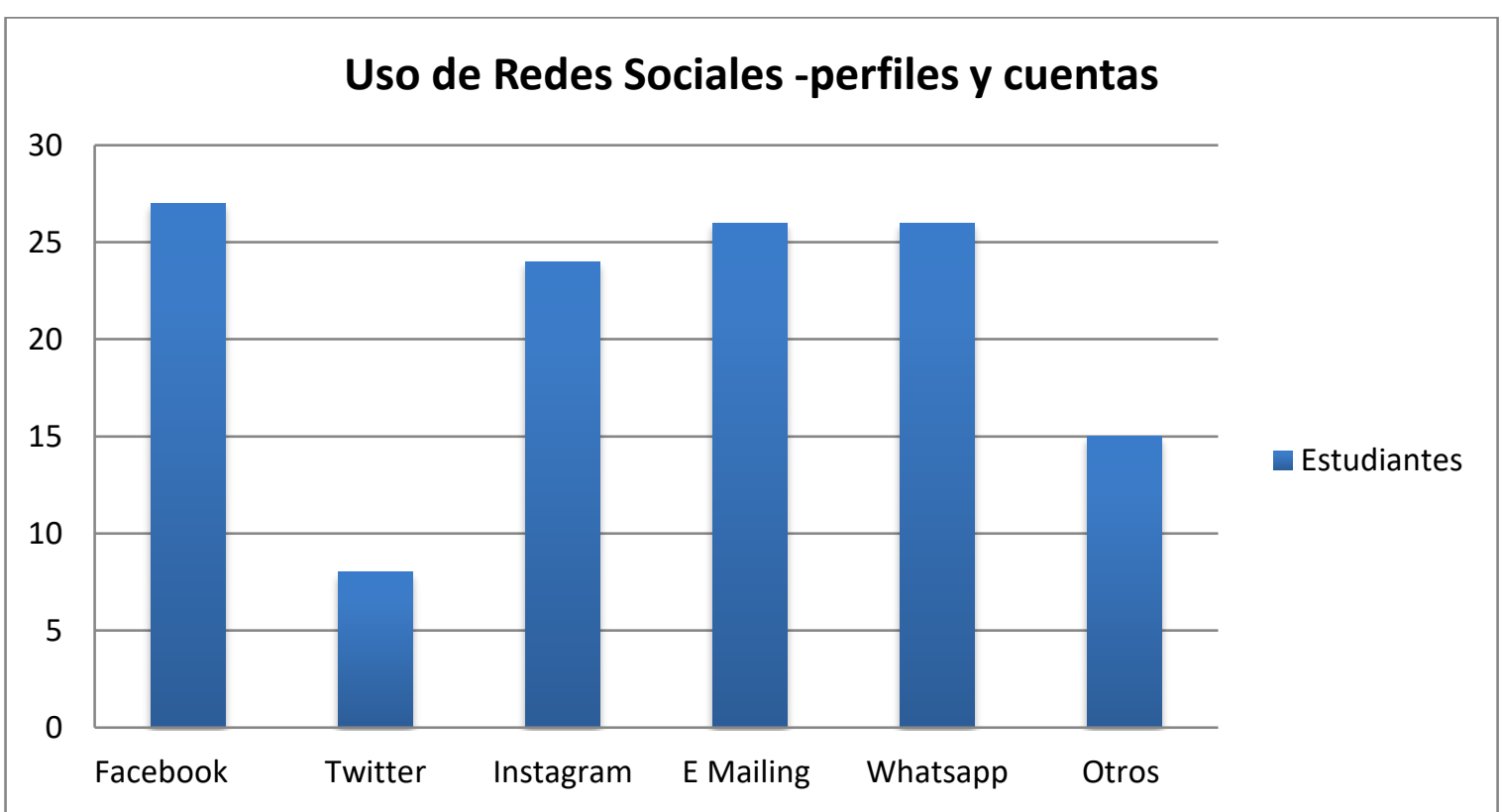

Ante la pregunta de qué forma utiliza usted aplicaciones tecnológicas, una mayoría indicó que utiliza videos o audios para aprender inglés mientras que una pequeña parte del grupo dijo casi no utilizarlo para compartir ideas o foros de discusión en línea. Lo anterior demuestra que para los estudiantes les es muy atractivo las herramientas audiovisuales para aprender. El gráfico 1 presenta esta información con más detalle. 
Uso del WhatsApp en Comunidades Interactivas de Aprendizaje: Perspectiva Estudiantil sobre Ejercicios de Pronunciación en la Clase de Inglés para Propósitos Específicos

Gráfico 3. Uso de aplicaciones tecnológicas

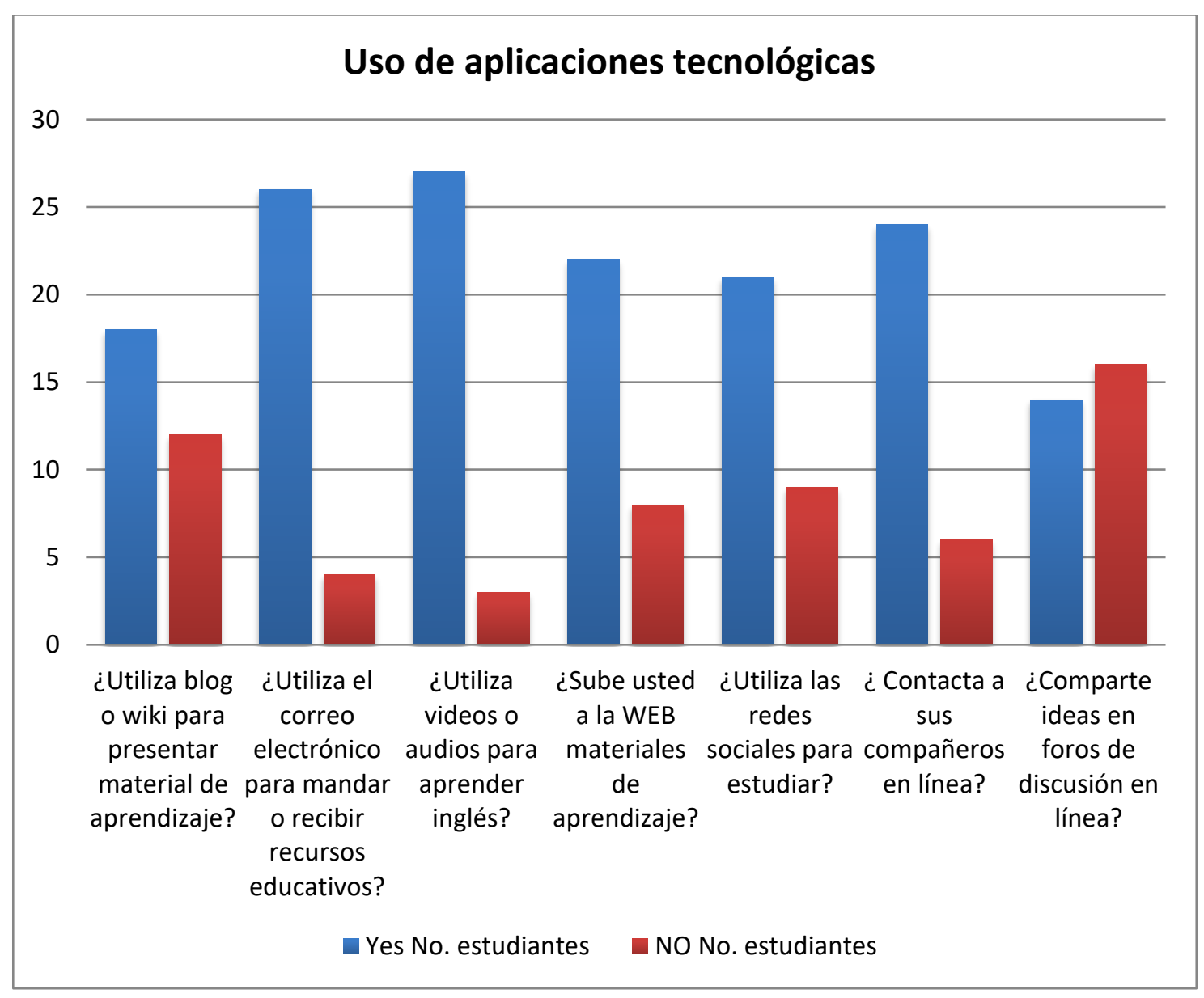

Una mayoría de los estudiantes manifestaron, en igual medida, ser más competentes al utilizar el whatsApp y navegar por Internet, lo que significa que los alumnos se sienten muy familiarizados con éstos y no tienen problema de echar mano de ellos para aprender. 
Gráfico 4: Qué tan competente es utilizando las aplicaciones para propósitos de Aprendizaje

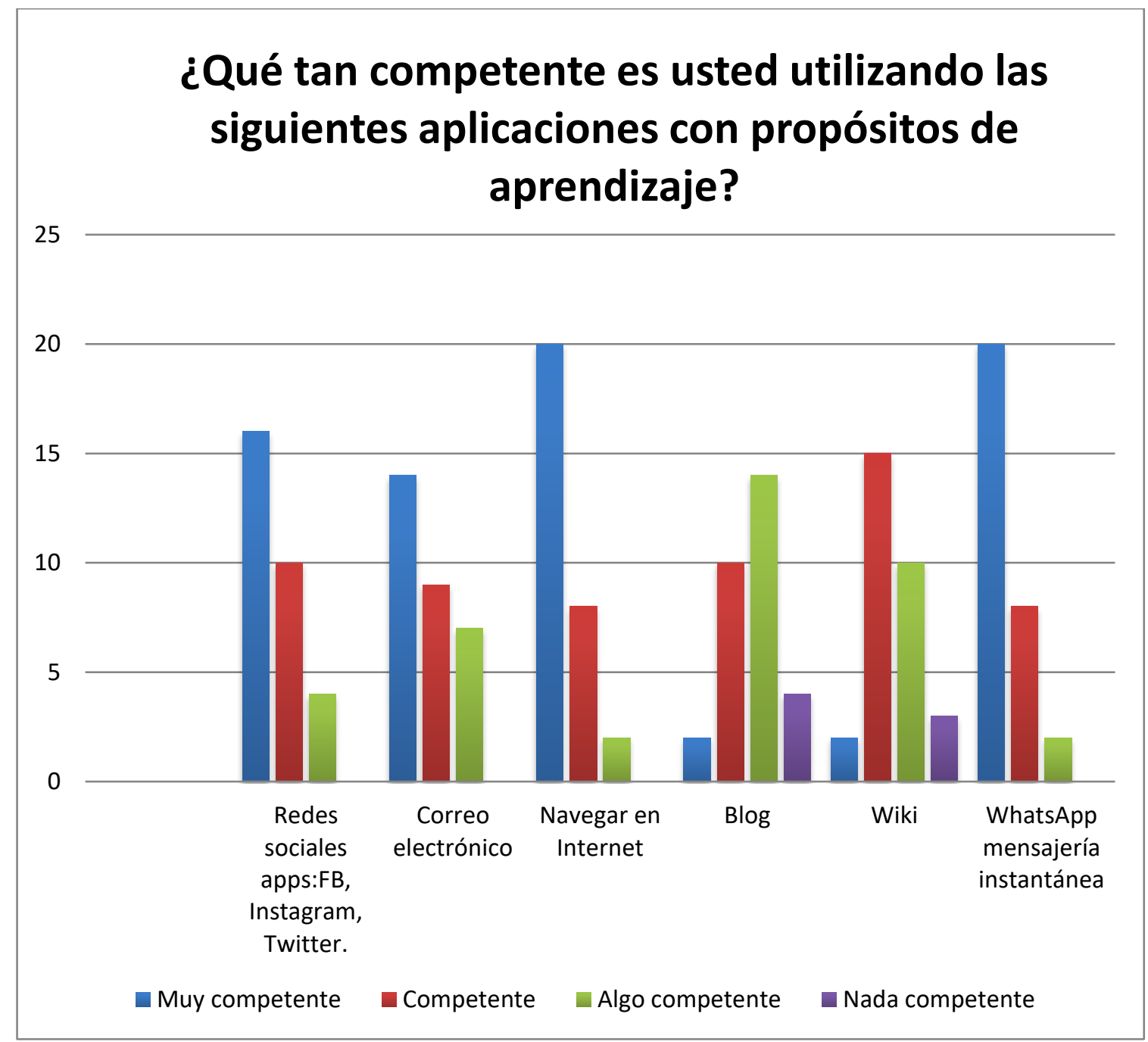

Al preguntarles la frecuencia en el uso de las diferentes aplicaciones para aprender, los estudiantes respondieron que utilizan más el navegar por Internet. Le sigue WhatsApp y muy cerca las redes sociales. Con esta información vemos que persiste la incidencia del uso del WhatsApp.Por otra parte, al preguntar sobre el uso del whatsApp en diferentes tareas académicas, las respuestas en general muestran una mayor tendencia a estar de acuerdo en que ésta aplicación es una ayuda para su proceso de aprendizaje. Llama la atención, por ejemplo, las respuestas a como el WhatsApp incrementa relaciones con amigos , o bien, como juega un papel preponderante como medio informativo del quehacer universitario. 
Uso del WhatsApp en Comunidades Interactivas de Aprendizaje: Perspectiva Estudiantil sobre Ejercicios de Pronunciación en la Clase de Inglés para Propósitos Específicos

En la tabla de abajo se muestran los aspectos cuestionados a los estudiantes, seguidos del gráfico que ilustra las respuestas a los mismos.

Tabla 3: El uso WhatsApp como herramienta de aprendizaje

\begin{tabular}{|c|c|c|c|c|c|c|}
\hline & Pregunta & $\begin{array}{l}\text { Totalmente } \\
\text { de acuerdo }\end{array}$ & $\begin{array}{c}\text { De } \\
\text { acuerdo }\end{array}$ & Indecisa & $\begin{array}{c}\text { En } \\
\text { desacuerdo }\end{array}$ & $\begin{array}{l}\text { Totalmente } \\
\text { en } \\
\text { desacuerdo }\end{array}$ \\
\hline $\begin{array}{l}\text { Creo que el WhatsApp es importante como medio } \\
\text { de aprendizaje, especialmente para compartir } \\
\text { información relacionada con Turismo. }\end{array}$ & 1 & 14 & 13 & 1 & 2 & \\
\hline $\begin{array}{l}\text { Con los chats del WhatsApp en la Universidad me } \\
\text { mantengo informada de las obligaciones en los } \\
\text { diferentes cursos. }\end{array}$ & 2 & 20 & 9 & 1 & & \\
\hline $\begin{array}{l}\text { Teniendo clases en WhatsApp me ayuda a } \\
\text { desarrollar las habilidades de trabajo en equipo, }\end{array}$ & 3 & 8 & 12 & 6 & 3 & \\
\hline $\begin{array}{l}\text { WhatsApp me habilita a copmpartir información y } \\
\text { conectar ideas con compañeros. }\end{array}$ & 4 & 17 & 12 & 1 & & \\
\hline $\begin{array}{l}\text { WhatsApp aumenta mi interés y motivación en } \\
\text { clase }\end{array}$ & 5 & 11 & 12 & 4 & 3 & \\
\hline $\begin{array}{l}\text { Etiqutando a mis compañeros en posts útiles me } \\
\text { ayuda a aprender mejor. }\end{array}$ & 6 & 8 & 13 & 8 & 1 & \\
\hline $\begin{array}{l}\text { WhatsApp incrementa mi particiación en el } \\
\text { aprendizaje. }\end{array}$ & 7 & 8 & 13 & 7 & 2 & \\
\hline $\begin{array}{l}\text { Siento que WhatsApp learning es una pérdida de } \\
\text { tiempo. }\end{array}$ & 8 & 6 & 8 & 5 & 11 & \\
\hline $\begin{array}{l}\text { Conversando en el WhatsApp me ayuda a } \\
\text { mantener las relaciones sociales. }\end{array}$ & 9 & 11 & 13 & 5 & 1 & \\
\hline $\begin{array}{l}\text { WhatsApp no incrementa mi autoestima porque } \\
\text { no puedo expresar mis pensamientos } \\
\text { abiertamente. }\end{array}$ & 10 & 5 & 4 & 8 & 9 & 4 \\
\hline $\begin{array}{l}\text { WhatsApp incrementa la comunicación con mis } \\
\text { amigos de manera más eficiente. }\end{array}$ & 11 & 17 & 12 & 1 & & \\
\hline $\begin{array}{l}\text { WhatsApp es nuevo para mí y no me siento } \\
\text { cómodo usándolo }\end{array}$ & 12 & & 3 & & 19 & 8 \\
\hline Me estresa el WhatsApp stresses & 13 & & 4 & 9 & 14 & 3 \\
\hline $\begin{array}{l}\text { Mis notas serían mejores si pudiera contactar a } \\
\text { mis profesores a través del WhatsApp fuera del } \\
\text { horario de clase. }\end{array}$ & 14 & 6 & 9 & 9 & 6 & \\
\hline $\begin{array}{l}\text { Me doy cuenta que el WhatsApp ha cambiado la } \\
\text { manera en que aprendo. }\end{array}$ & 15 & 1 & 9 & 13 & 6 & 1 \\
\hline $\begin{array}{l}\text { WhatsApp no me ayuda a mi aprendizaje porque } \\
\text { no es fácil de usar. }\end{array}$ & 16 & 1 & 4 & & 14 & 11 \\
\hline $\begin{array}{l}\text { WhatsApp me ayuda a comprender conceptos de } \\
\text { manera más eficiente. }\end{array}$ & 17 & 4 & 12 & 5 & 7 & 2 \\
\hline $\begin{array}{l}\text { WhatsApp no es efectivo para obtener un buen } \\
\text { aprendizaje porque crea problemas técnicos. }\end{array}$ & 18 & & 1 & 9 & 13 & 7 \\
\hline
\end{tabular}


Gráfico 5: El uso del WhatsApp como herramienta de Aprendizaje

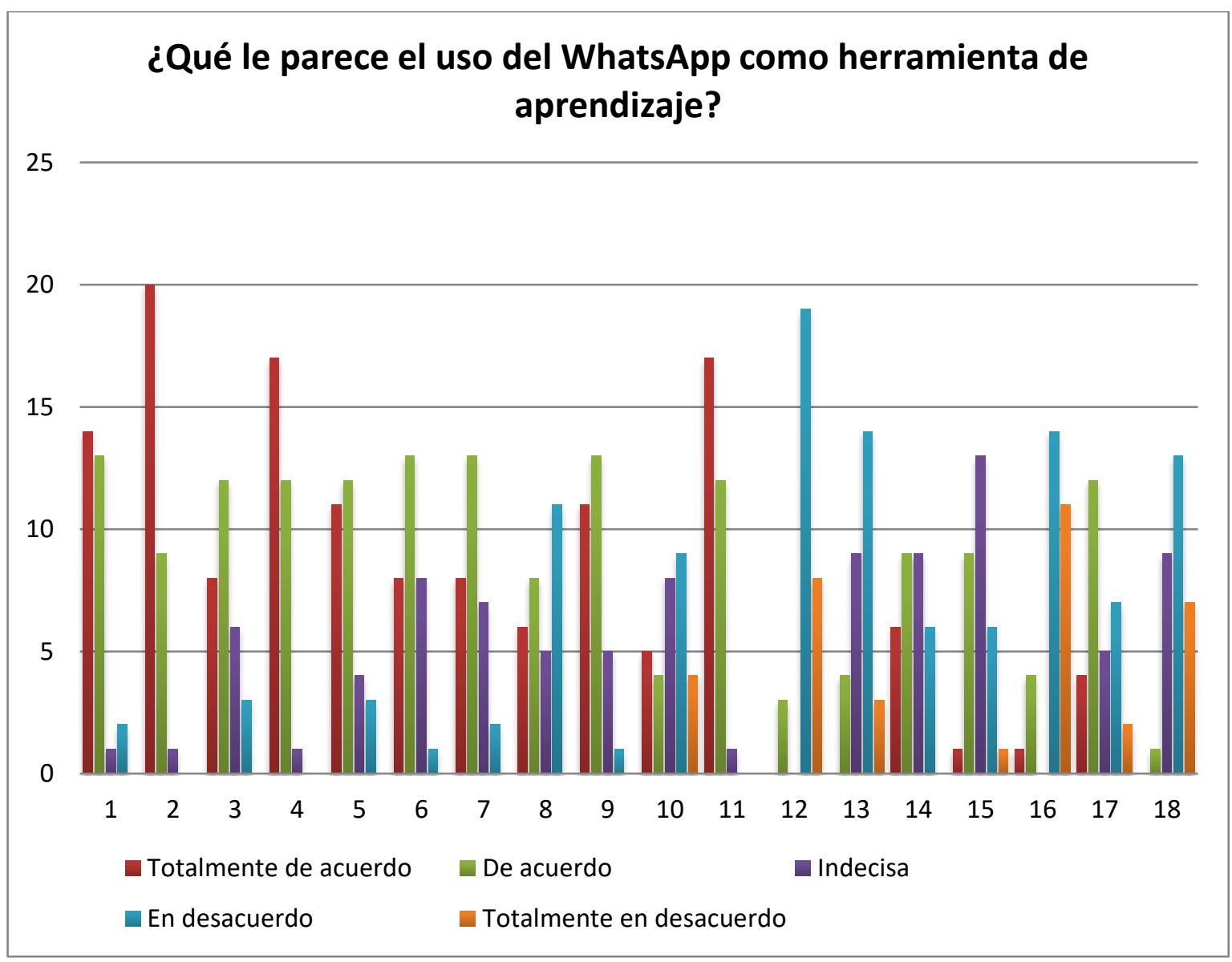

Las respuestas de opinión demuestran también mucha apertura sobre el uso del whatsApp para fines académicos, haciendo la aclaración que su buen uso y aceptación dependerá de la responsabilidad de cada quien al momento de utilizarlo o bien que puede ser un distractor al recibir otros chats o informaciones al momento de estar trabajando o estudiando.

\subsection{Resultados y Conclusiones}

En nuestro contexto educativo costarricense el uso de dispositivos móviles con propósitos académicos y como parte de la mediación del aprendizaje del idioma Inglés es aún limitado. En muchos países Europeos y especialmente en España, ha habido un gran interés en la incorporación de dispositivos móviles y ya son muchos los docentes de diferentes áreas del saber que día a día ven en los teléfonos inteligentes una oportunidad, un instrumento para 
Uso del WhatsApp en Comunidades Interactivas de Aprendizaje: Perspectiva Estudiantil sobre Ejercicios de Pronunciación en la Clase de Inglés para Propósitos Específicos

mediar los aprendizajes. Aunque en Costa Rica ha habido un incremento en la implementación de las TICS en la mediación de los aprendizajes, el uso y la implementación de dispositivos móviles en la mediación de los aprendizajes es aún muy limitado. Todos concordamos en el hecho de que los teléfonos celulares inteligentes han cambiado radicalmente nuestras sociedades, quien hubiese atinado hace décadas atrás que un dispositivo móvil serviría para realizar innumerables actividades influyendo y transformado radicalmente nuestra cotidianidad en tan importantes maneras. Estos dispositivos han sido ampliamente aceptados en otros países por ser herramientas con un gran atractivo, sus características inteligentes acceso a internet, cámara, grabación de audio e imagen y las variadas aplicaciones a las que se puede acceder los hace aún más atractivos. Por lo que ya son muchos los investigadores, académicos y docentes que los incorporan en la mediación académica y estos son generalmente bien aceptados en los procesos de aprendizaje.

En nuestro caso especifico nuestros estudiantes se mostraron positivos y abiertos ante la oportunidad de implementar el WhatsApp como herramienta para mediar practicas de pronunciación. Ellos en su mayoría enfatizaron que es una buena idea para facilitar el aprendizaje ya que se pueden beneficiar de diferentes maneras y ante esto puntualizaron que esta aplicación en el aprendizaje es popular, es gratis, casi todos los estudiantes la tienen, es una buena idea para compartir información y llevar a cabo discusiones académicas. Además, se fortalecen relaciones sociales en el curso pues se comparte en tiempo real con una herramienta que es de fácil manejo y que representa mayor atractivo interactivo a la hora de interactuar entre pares. Esta interacción entre compañeros y profesor mediante el grupo WhatsApp resulta divertida, y entretenida bajando los niveles de ansiedad que se genera al aprender y producir un idioma extranjero. interactuar con compañeros y profesor.

Algunas de las preocupaciones expuestas por los estudiantes ante la implementación de esta aplicación en el aula es el hecho de no contar con acceso a internet y que este se sobre use en clases, saturándolos con actividades y tareas académicas. Entre las sugerencias a resaltar, los estudiantes mencionaron incluir en los chats de WhatsApp links de otras aplicaciones como "You Tube", juegos interactivos, canciones, fotos, videos, presentaciones y ejercicios interactivos con nota. Igualmente, sugieren realizar los chats con comunidades pequeñas y no saturarlo con muchos mensajes para no sentirse "invadidos" con mucha información. Insisten en un uso responsable y que se tome con seriedad y no para enviar información que no se relacione con objetivos de aprendizaje.

\section{Bibliografia}

ALFARO, G. (1996). Evaluación cualitativa: técnicas y estrategias. San José, Costa Rica: EUNA.

BARHOUMI, CH, (2015) 'The effectiveness of whatsapp mobile learning activities guided by activity theory on students' knowledge management:. Contemporary educational technology, Vol.6 2015 (3), pp.221-238 https://eric.ed.gov/?id=EJ1105764

(Consulta : 22 Marzo de 2017)

BORDAS, M. Y CABRERA F. (2001). "Estrategias de evaluación de los aprendizajes centrados en el proceso". Revista Española de Pedagogía. Año LIX, enero abril , pp.25 - 48.

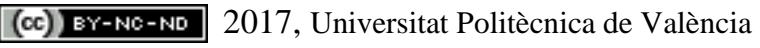

Congreso IN-RED (2017) 
( Consulta : 25 Marzo de 2017)

BRAZUELO, F., GALLEGO, D. (2011). Mobile Learning. Los dispositivos móviles como recurso educativo. Sevilla: Editorial MAD, S.L

CALDERÓ, R. (2014). El uso del teléfono móvil y de la aplicación WhatsApp para el aprendizaje de verbos frasales ingleses en alumnos de nivel intermedio. Trabajo Final de Master. La Rioja: Universidad Internacional de La Rioja http://reunir.unir.net/bitstream/handle/123456789/2639/caldero\%20gene.pdf?sequence=1\& isAllowed=y (Consulta : 20 Marzo de 2017)

DALE, P., Y POMS, L., (2005). English Pronunciation Made Simple. New York : Longman

GUTIÉRREZ, M., GIBERT, M.I., TRIANA, I., GIMENO, A., APPEL, C. \& HOPKINS, J. (2013). “Improving learners' reading skills through instant short messages: A sample study using WhatsApp." University of Ulster. En: worldcall: sustainability and computer-assisted language learning Conference.Glasgow pp.80-84. Disponible en https://www.researchgate.net/publication/255718202_Improving_learners\%27_reading_ski 1ls_through_instant_short_messages_a_sample_study_using_WhatsApp

HIDALGO, G. (2013). “Uso del teléfono móvil: posibilidades didácticas y riesgos en los jóvenes" Trabajo Final de Master. Almeria: Universidad Almeria.

http://repositorio.ual.es/bitstream/handle/10835/2353/Trabajo.pdf?sequence=1\&isAllowed

( Consulta : 20 Marzo de 2017)

HOCKLY, N., DUDENEY, G. (2014). Going mobile: Teaching with hand-held devices. Estados Unidos:Delta Publishing.

IRUELA, G. (2007). “¿Qué es la pronunciación ?’” RedEle Revista Electrónica Didáctica. Número 9.

( Consulta : 25 Marzo de 2017)

KOPER, R. (2009). Learning Network Services for Professional Development. Berlin: Heidelberg: Springer.

THORNTON, P., HOUSER, C. (2005). "Using mobile phones in English education in Japan. en Journal of Computer Assisted Learning, 21,217-228. http://dx.doi.org/10.1111/j.1365-2729.2005.00129.x

( Consulta : 20 Marzo de 2017)

WEI , M. (2006). “A Literature Review on Strategies for Teaching Pronunciation". Maryland : University of Maryland at College Park.

http://files.eric.ed.gov/fulltext/ED491566.pdf

( Consulta : 25 Marzo de 2017) 